\title{
Additive Induced Formation of Ultrathin Sodium Chloride Needle Crystals
}

\author{
Eleanor R. Townsend, ${ }^{\dagger}$ Willem J. P. van Enckevort, ${ }^{*}{ }^{\dagger}$ (i) Paul Tinnemans, ${ }^{\dagger}$ Melian A. R. Blijlevens, ${ }^{\dagger}$ \\ Jan A. M. Meijer, ${ }^{\ddagger}$ and Elias Vlieg ${ }^{\dagger}$ \\ ${ }^{\dagger}$ Radboud University, Institute for Molecules and Materials, 6500 GL Nijmegen, The Netherlands \\ ${ }^{\ddagger}$ AkzoNobel Industrial Chemicals, RD\&I Salt, 7418 AJ Deventer, The Netherlands
}

Supporting Information

ABSTRACT: A multitude of ultrathin crystal needles are formed during the evaporation of saturated aqueous $\mathrm{NaCl}$ solution droplets in the presence of amide containing additives. The needles are as small as $300 \mathrm{~nm}$ wide and $100-1000 \mu \mathrm{m}$ in length. Heating experiments, X-ray diffraction, and energy dispersive X-ray spectroscopy showed that the needles are cubic sodium chloride crystals with the needle length direction pointing toward [100]. This shape, not expected for the $4 \overline{3} \mathrm{~m}$ point group symmetry of $\mathrm{NaCl}$, has been explained using a model, based on tip formation by initial
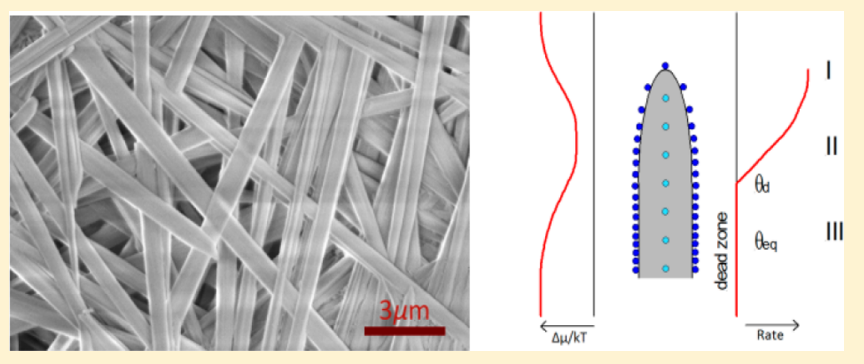
morphological instability followed by time dependent adsorption of additive molecules blocking the growth of the needle side faces. The latter also suppresses side branch formation, which normally occurs for dendrite growth.

\section{INTRODUCTION}

Much work has been done into the influence of additives on the habit of sodium chloride crystals. The earliest known example was published by Rome de l'Isle in $1783,{ }^{1}$ when he found that the addition of urea to a brine solution causes the development of $\{111\}$ facets on the resultant crystals. Since then, there have been many additives found which have an effect on either the morphology or the growth of salt. For example, the addition of glycine to a brine solution has been shown to elicit $\{110\}$ facets on the crystals ${ }^{2}$ and formamide and several chromium and lead compounds have been shown to elicit $\{111\}$ facets. $^{3-5}$ The stability of the polar $\{111\}$ faces in the presence of formamide and $\mathrm{Cd}^{2+}$ has been explained by Radenovic et al., ${ }^{6,7}$ on the basis of surface X-ray diffraction measurements. The most widely utilized additive for $\mathrm{NaCl}$ currently is ferrocyanide, which causes a morphology change from cubic to dendritic, and has a positive effect on preventing caking of the material and is therefore very industrially relevant.

It has been mentioned as far back as in $1961^{8}$ that another additive, nitrilotriacetamide (NTAA), has an anticaking effect on sodium chloride at concentrations as low as $1 \times 10^{-4} \%(\mathrm{w} / \mathrm{w})$. Its anticaking effectivity was also mentioned in the 1966 work by L. Phoenix ${ }^{9}$ and the 1975 work by Sarig et al. ${ }^{10}$ The latter investigation described in detail the observed habit modification of $\mathrm{NaCl}$ crystals in the presence of $50 \mathrm{ppm}$ of NTAA, presenting skeleton cubic morphology. A brief model was also presented, showing the expected binding points of the NTAA molecule on the $\mathrm{NaCl}\{100\}$ surface. The follow-up article to this investigation $^{11}$ showed that at higher concentrations of NTAA (100-500 ppm), the $\mathrm{NaCl}$ habit was further modified to display dendritic growth.
Current uses of NTAA and its sister compounds, methylglycine diacetamide (MGDA) and glutamic acid diacetamide (GLDA), are as chelating agents, Dissolvine in their sodium salt forms. NTAA has also been reported to reduce sodium chloride deposition in the drilling of oil wells. ${ }^{12,13}$ However, there is no current usage of NTAA or other branched amides in the salt anticaking industry.

The formation of sodium chloride needles was first mentioned in a 1932 note by Tauber and Kleiner ${ }^{14}$ which stated that sodium chloride needles are formed when suspending an undersaturated solution in a colloidion bag (a membrane used to filter or concentrate substances using pressure) and allowing the solution to slowly evaporate. In 1933, Hinegartner ${ }^{15}$ also observed sodium chloride needles but this time growing on a silica gel substrate. Sears ${ }^{16}$ also observed the formation of needle crystallites (thus termed whiskers) from bulk crystals including $\mathrm{NaCl}$, with a more in-depth investigation being performed by Shichiri and collaborators. ${ }^{17-19}$ Specifically, needle formation of $\mathrm{NaCl}$ in the presence of NTAA was mentioned by van Dammevan Weele. ${ }^{20}$ Also, using the technique of vapor growth, Noorduin observed an unusual formation of sodium chloride with extreme elongation in the $\langle 100\rangle$ direction. ${ }^{21}$

In our previous work, we have described the technique of creeping, in which a droplet of solution containing brine and additive is allowed to evaporate, and the resulting pattern is examined to determine the effectivity of the additive in the solution. In general, the greater the amount of creeping from the

Received: August 21, 2017

Revised: December 8, 2017

Published: December 8, 2017 
edges of the droplet, the more effective the anticaking agent is for sodium chloride. ${ }^{22}$ Using creeping, we have shown that the addition of large amounts $(1 \%(\mathrm{w} / \mathrm{w}))$ of nitrilotriacetamide and methylglycine diacetamide to a saturated brine solution causes the formation of needle-shaped sodium chloride crystals when allowed to evaporate on a glass substrate. ${ }^{22,23} \mathrm{~A}$ very compact evaporation pattern is observed, with many needles layered on top of each other. However, it is not known what is the mechanism behind the formation of these needle crystals. Using amide compounds of similar structure (Figure 1), we aim to characterize this needle growth using an experimental approach, complemented with a theoretical model to describe our observations.

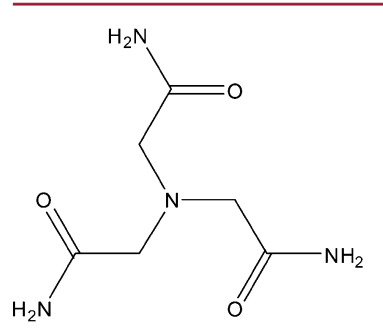

Nitrilotriacetamide (NTAA)

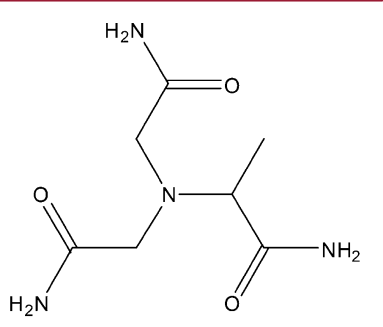

Methylglycine diacetamide (MGDA)

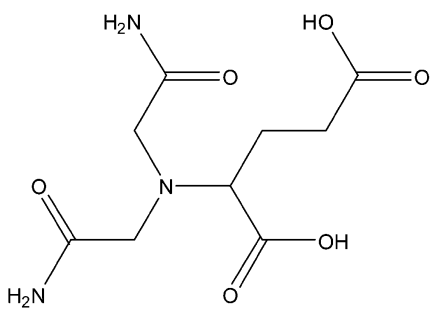

Glutamic acid diacetamide (GLDA)

Figure 1. Structures of the amide compounds used in this investigation.

\section{METHODS}

Nitrilotriacetamide has been widely reported in the literature in relation to sodium chloride, as described in the introduction. These observations were confirmed by our more recent work, ${ }^{23}$ which showed it causes a habit modification of the crystals at high concentrations in addition to being an effective anticaking agent for $\mathrm{NaCl}$. The same effect has been observed for the sister molecule, methylglycine diacetamide. ${ }^{22}$

The third compound used in this investigation is the related compound, glutamic acid diacetamide. This is unreported in the literature in free amide form, but the sodium salt is used currently as a chelating agent.

The concentrations of the additives in solution used for each experiment were set at specific weight percentages with respect to the solution to ensure fair testing, with each additive containing brine solution being in the $\mathrm{pH}$ range of 5-6. The exception to this is the GLDA containing brine solution, which has a higher $\mathrm{pH}$ of 3.5, due to the presence of the two acid groups in the molecule. The concentrations used in the experiments were in a range between $0.1 \%(\mathrm{w} / \mathrm{w})$ and $4 \%$ (w/w).

Creeping Tests. A solution was made using saturated brine and a certain percentage of the chosen additive at ambient temperature (20$25^{\circ} \mathrm{C}$ ). The solution was filtered using a syringe fitted to a $0.45 \mu \mathrm{m}$ Pall GHP Acrodisc filter, and then dropped onto a glass microscope slide using a micropipette. These slides were cleaned beforehand using EtOH and dried using a dust-free tissue to minimize external nucleation effects.
The amount of solution in each droplet varied from 5 to $10 \mu \mathrm{L}$. The droplets were allowed to evaporate in a temperature and humidity controlled climate chamber at $20{ }^{\circ} \mathrm{C}$ and $50 \%$ relative humidity. The resulting crystals were imaged using a Leica DMRX optical microscope, a FEI Phenom scanning electron microscope and a JEOL 6330 field emission scanning electron microscope. The optical microscope was also used to follow the creeping process in situ at ambient conditions $\left(\sim 20^{\circ} \mathrm{C}\right.$ and $\left.\sim 50 \% \mathrm{RH}\right)$.

Scanning Electron Microscopy (SEM). In order to view our samples at a sufficiently high magnification with a high depth of field, we used scanning electron microscopy (SEM). The creeping patterns were dried in a climate controlled chamber at $20{ }^{\circ} \mathrm{C}$ and $50 \%$ relative humidity and then sputtered with $\mathrm{Au}-\mathrm{Pd}$ using a Cressington 208HR sputter coater. The sample was left with a coating in the range of $0.5-3$ $\mathrm{nm}$ in thickness. The samples were imaged in backscattering mode at 5 $\mathrm{kV}$ for the FEI SEM and in secondary electron mode at $12 \mathrm{kV}$ for the JEOL 6330.

Energy Dispersive X-ray Spectroscopy (EDX). Mapping by EDX was used to determine the two-dimensional elemental composition of the sample surface. This was performed using a Zeiss Sigma 300 scanning electron microscope, coupled with a Bruker energy-dispersive $\mathrm{X}$-ray spectrometer.

Solution Crystal Growth and Single-Crystal X-ray Diffraction. The salt used in this investigation is Sanal P, an AkzoNobel product of 99.99\% purity, and the additives used were obtained at a commercial grade from AkzoNobel, containing possible side products. Therefore, each compound was recrystallized out of solution by slow evaporation at a constant temperature and humidity $\left(20^{\circ} \mathrm{C}\right.$ and $50 \%$ R.H.). The resulting crystals were then analyzed using single crystal X-ray diffraction to determine their compositions and structures. The crystal structures of MGDA and GLDA were previously unknown and are submitted to the Crystal Structural Database under codes CCDC 1533349 and CCDC 1533348 respectively. The $\mathrm{NaCl}$ needles obtained by the creeping experiments were examined by powder and single crystal X-ray diffraction. In powder $\mathrm{X}$-ray diffraction experiments $\mathrm{Cu} \mathrm{K} \alpha$ radiation was used, and in single crystal measurements, Mo radiation was used.

\section{EXPERIMENTAL RESULTS AND DISCUSSION}

At low magnification, on the addition of $1 \%(\mathrm{w} / \mathrm{w})$ NTAA/MGDA/ GLDA to a saturated brine solution, the resulting evaporation pattern consists of a dense formation of crystallites with no evidence of significant cubic crystal growth (see Figure $2 a$ ). From Figure $2 b$, it is
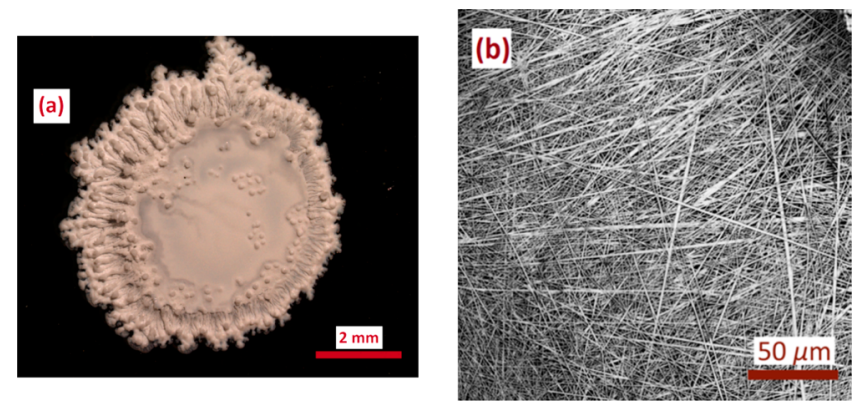

Figure 2. (a) An optical overview image of a dried droplet of saturated brine with $1 \%(\mathrm{w} / \mathrm{w})$ NTAA, (b) the crystal pattern from the center of the droplet observed at higher magnification using SEM.

evident that this formation is entirely filled with ultrathin needle-like crystallites with no definitive orientation. As described in our previous work, ${ }^{22}$ these needles form as a result of a lower surface free energy and an increased supersaturation, both of which promote extremely fast and numerous three-dimensional (3D) nucleation. In situ recording of the crystal growth process using optical microscopy at high magnification showed needle growth rates of approximately $10 \mu \mathrm{m} / \mathrm{s}$. This can be seen in the Supporting Information Movie S1.

To estimate the supersaturation at which the crystals first begin to nucleate from a saturated brine droplet with $1 \%(\mathrm{w} / \mathrm{w})$ NTAA as 
additive, an evaporating droplet on a glass substrate was weighted in situ using a precision balance. From the change in weight of the droplet when the first crystallites appeared as imaged using optical microscopy, we arrived at an approximate average figure of $\Delta \mu / k T \approx 0.16$. This surpasses the value measured for pure brine, ${ }^{23} \Delta \mu / k T \approx 0.06$, which indicates a large supersaturation dead zone before nucleation occurs.

Composition of Needles. Because of the relatively large amounts of additive used in these experiments, it was important to check the chemical composition of the needles, to determine if they consist of sodium chloride, or the added amide compound.

The melting point of $\mathrm{NaCl}$ is high as it is an ionic compound, with a value of $801{ }^{\circ} \mathrm{C}$. In comparison, the measured melting points for the additive compounds are much lower, being in the range of $160-200^{\circ} \mathrm{C}$. These were determined using differential scanning calorimetry (DSC) measurements and are displayed in Table 1 . The error presented is from the technical specifications of the DSC instrument (Mettler Toledo DSC 822e).

\section{Table 1. Additives Used and Their Melting Points}

$\begin{array}{cc}\text { compound name } & \text { melting point }\left({ }^{\circ} \mathrm{C} \pm 0.2{ }^{\circ} \mathrm{C}\right) \\ \text { NTAA } & 194.4 \\ \text { MGDA } & 176.1 \\ \text { GLDA } & 167.4\end{array}$

Using a temperature controlled cell, attached to an optical microscope, the sample was heated from 20 to $400{ }^{\circ} \mathrm{C}$ at a rate of 20 ${ }^{\circ} \mathrm{C} / \mathrm{min}$. The sample was examined in situ by this microscopy during heating and a posteriori using SEM for evidence of decomposition of the crystallite microstructures. There was no evidence that the needle crystallites decomposed, apart from a slight black coating over the sample. This is a strong indication that the needles are indeed composed of $\mathrm{NaCl}$ and not the amide additive. It is likely that the decoloration on the top of the sample is due to the decomposition of the amide compounds which coat the needles. A post experiment SEM image is shown in Figure 3.

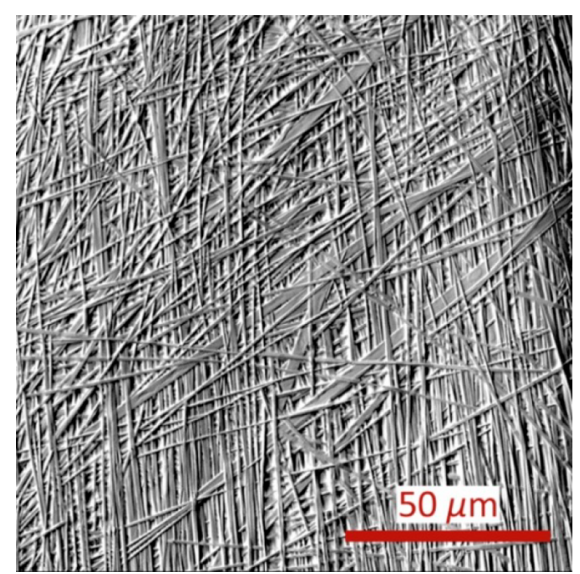

Figure 3. SEM image of needle pattern from a saturated solution of brine with $1 \%(\mathrm{w} / \mathrm{w})$ NTAA additive, after heating to $400{ }^{\circ} \mathrm{C}$.

It was not possible to take a pre-experiment SEM image of this sample, due to the necessity of Au sputtering to make the sample conductive, but the crystallites can be favorably compared with other samples, and we conclude that there was little change in the overall needle structure, which shows a pattern very similar to that without heat treatment (Figure 2b).

In order to confirm our findings, powder X-ray diffraction (PXRD) measurements were taken of the samples $(\mathrm{NaCl}$ with $1 \%(\mathrm{w} / \mathrm{w})$ amide additive) and compared with theoretical and measured patterns of pure $\mathrm{NaCl}$ and pure amide. These are shown in Figure 4. The powder pattern of NTAA was taken from the Crystal Structural Database (reference code: VUSMOL), and those of MGDA and GLDA were taken from the theoretical powder patterns calculated from the single crystal measurements described earlier.

It is evident from these results that the diffraction peaks observed for the needles and the pure $\mathrm{NaCl}$ match, whereas those for the pure amides do not. There is one extra peak noticeable in the PXRD for NTAA additive at $32^{\circ}$, and it is not known exactly what this corresponds to, but it is possible that it is a small amide peak.

To obtain a final confirmation that the needles indeed consist of $\mathrm{NaCl}$, an EDX measurement was performed showing that the crystals indeed consist of $\mathrm{Na}$ and $\mathrm{Cl}$; see Figure 5. The measurement also showed the presence of $\mathrm{Au}$ as the sample had to be conductively sputtered. As is also evident from the graph, there is very little nitrogen present, and there is also there is also only a very low level of carbon, at $0.277 \mathrm{keV}$, indicating that the needles do not consist of amide.

To obtain information on the crystallographic orientation of the needles, we selected a somewhat thicker needle from the NTAA sample and applied single crystal X-ray diffraction to it. This showed that the crystallographic directions of the top and side faces of the needle crystals are the $\langle 100\rangle$ directions. This is illustrated in Figure 6.

Properties of Needles. To obtain more detailed information on needle size and shape, high magnification observations using field emission SEM are performed. Some results are displayed in Figure 7. The needles show no preferred orientation and form a fiber-like network across the substrate, filling all available space. The needles are extremely thin, with a width varying from 0.3 to $2 \mu \mathrm{m}$ and a length ranging from $10^{2}-10^{3} \mu \mathrm{m}$. Except for the case of GLDA (Figure 7f), no evidence of side branching was found. There is also no evidence of any cubic crystal growth across the growth pattern. This is in contrast to the growth experiments in the absence of additive, which only produce well-faceted cubes. $^{22}$

Close inspection of the SEM images show that the needles have a rounded, nonfaceted tip. Some needles have a ridge lengthways down the center. The side facets of the needles have a tendency toward cubic morphology, but as growth in one direction is extremely fast, the final form is an extremely elongated cube. This confirms that the ultrathin needle axis direction is $\langle 100\rangle$ as was also observed for the thicker needle in Figure 6.

Dependence of Additive Concentration on Needle Formation. A series of creeping experiments were carried out at concentrations lower and higher than that of $1 \%(\mathrm{w} / \mathrm{w})$ NTAA to determine the necessary additive concentration to obtain needle crystallites.

It was observed at concentrations of $0.2 \%(\mathrm{w} / \mathrm{w})$ NTAA and lower that needle crystallites were no longer present in the creeping pattern; only a tendency toward cubic growth was found. SEM images of a series of creeping experiments with decreasing levels of additive are shown in Figure 8.

Similarly, at concentrations of $2 \%(\mathrm{w} / \mathrm{w})$ NTAA, the needle crystallites begin to disappear, making way for dendritic growth. At an additive concentration of $4 \%(\mathrm{w} / \mathrm{w})$ NTAA, there are no needle crystallites anymore. The $\mathrm{NaCl}$ crystals observed at these concentrations of additive have high levels of morphological instability with no clear $\{100\}$ facets, as is shown in the SEM images of Figure 9, so needles are only formed for additive concentrations ranging from 0.75 to $\sim 1.5 \%$ (w/ w) NTAA.

\section{MECHANISM OF NEEDLE FORMATION}

Principle. The formation of thin, elongated $\mathrm{NaCl}$ needles is not expected for the cubic $4 \overline{3} \mathrm{~m}$ point group symmetry of $\mathrm{NaCl}$. The main question that arises is why does the (100) top face grow fast, while the symmetry equivalent $(010) /(0 \overline{1} 0)$ and $(001) /(00 \overline{1})$ side faces of the cubic crystals do not grow at all. As we will argue below, we believe this is caused by tip formation induced by morphological instability followed by time-dependent adsorption of the additives blocking the growth of the needle side faces. In our case the anisotropic growth of the needles is not explained by the presence of dislocations parallel to the needle 


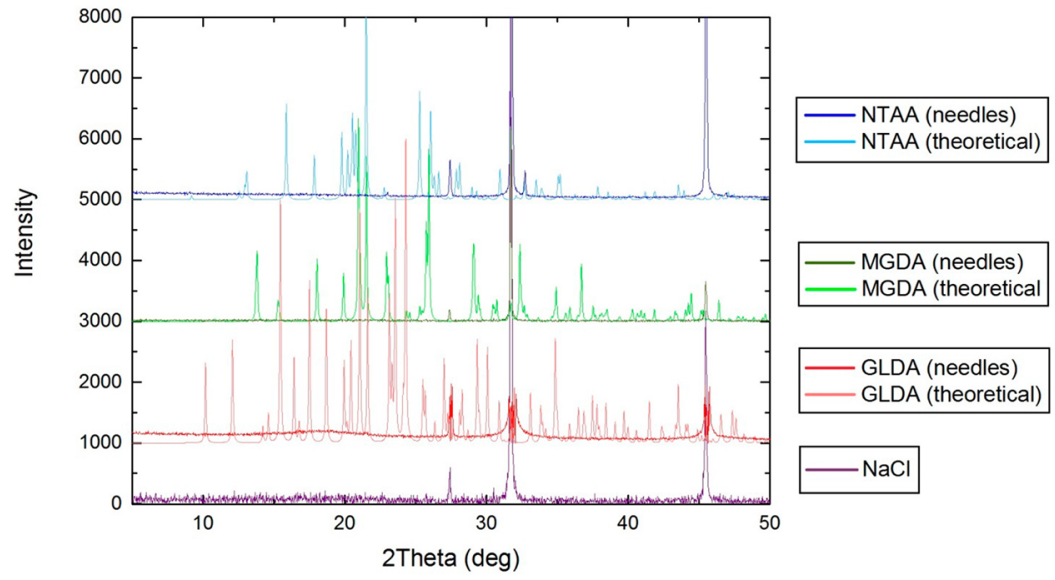

Figure 4. PXRD patterns comparing pure $\mathrm{NaCl}$, pure amide, and needle crystallites for $\mathrm{NTAA}, \mathrm{MGDA}$, and GLDA. $\mathrm{NaCl}$ peaks are observed at $27^{\circ}, 32^{\circ}$, and $41^{\circ}$.

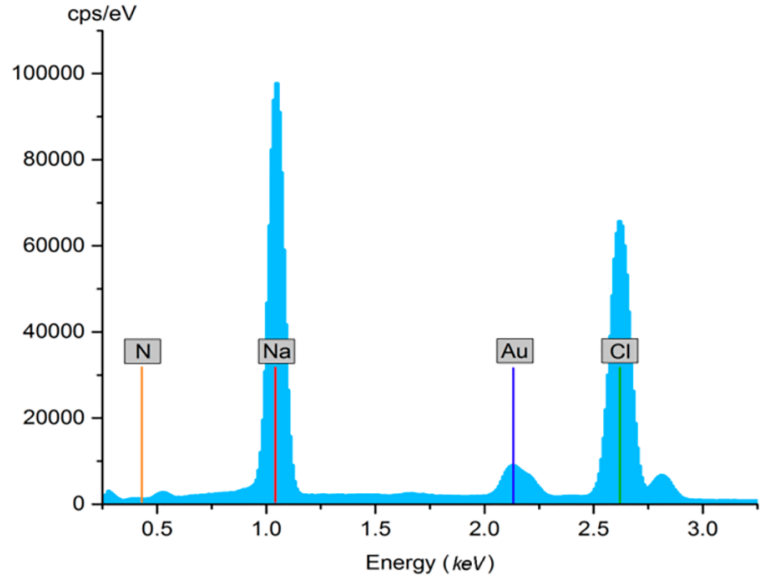

Figure 5. EDX graph showing the expected and measured peaks for $\mathrm{Na}$, $\mathrm{Cl}, \mathrm{N}$, and $\mathrm{Au}$.

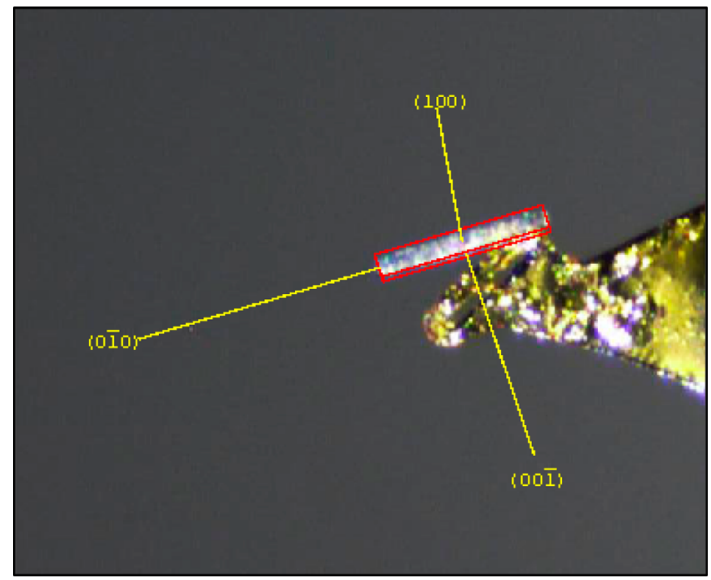

Figure 6. Microscope image of single needle crystal with crystallographic planes indicated, as determined through single crystal X-ray diffraction. The dimensions of the crystal are $0.014 \times 0.032 \times 0.326$ $\mathrm{mm}^{3}$.

axis and their absence directing toward the side faces, as the tips are not faceted, so spiral growth does not occur. Further, no reentrant corner was observed at the needle tips, which excludes enhanced growth by the twin plane re-entrant edge (TPRE) mechanism. ${ }^{24-26}$ Further, neither in situ microscopic nor X-ray
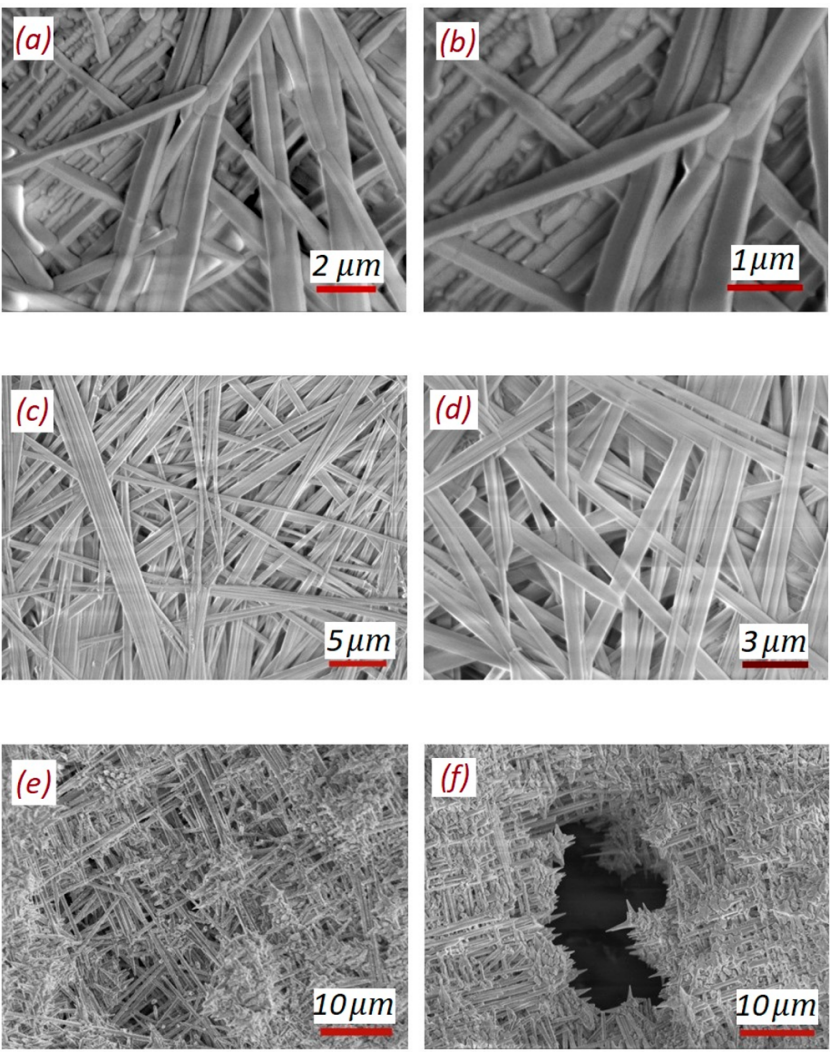

Figure 7. Detailed SEM views of needle shaped crystals obtained from a saturated brine solution containing $(\mathrm{a}-\mathrm{b}) 1 \%(\mathrm{w} / \mathrm{w})$ NTAA, $(\mathrm{c}-\mathrm{d}) 1 \%$ (w/w) MGDA, (e-f) 1\% (w/w) GLDA.

evidence was found for recrystallization from an unknown metastable form of $\mathrm{NaCl}$ to cubic $f c c \mathrm{NaCl}$. Finally, morphological instability does not fully explain the development of the needles as follows from the fact that the needles often do not show a preferred direction of growth induced by a gradient in supersaturation, but are crisscrossed. Moreover, no branching was observed, which is typical for morphological instability.

In our attempt to explain the formation of the needles, we were inspired by the experiments by Land et al..$^{27}$ and the theoretical model of Muira ${ }^{28}$ for stepped surfaces and an earlier study by one of the authors for roughened faces. ${ }^{29}$ Following Muira's model ${ }^{28}$ and the earlier work by Cabrera and Vermileya ${ }^{30}$ steps on a 

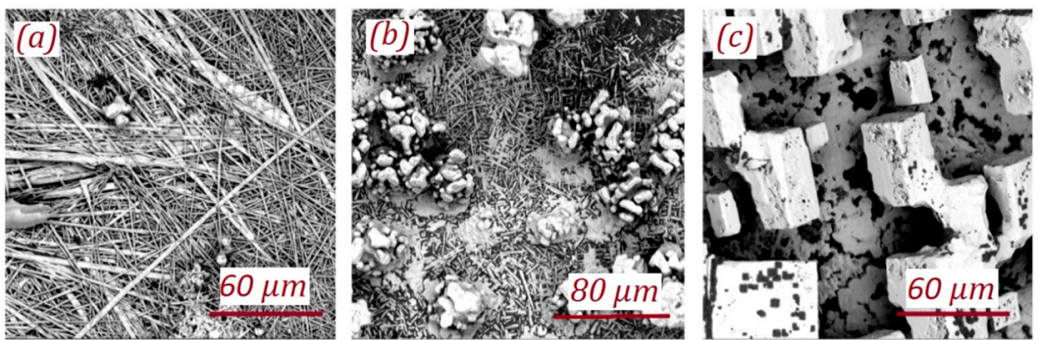

Figure 8. Detailed SEM views of needle-shaped crystals obtained from a saturated brine solution containing (a) $0.75 \%(w / w)$ NTAA, (b) $0.5 \%$ (w/w) NTAA, and (c) $0.25 \%(w / w)$ NTAA.
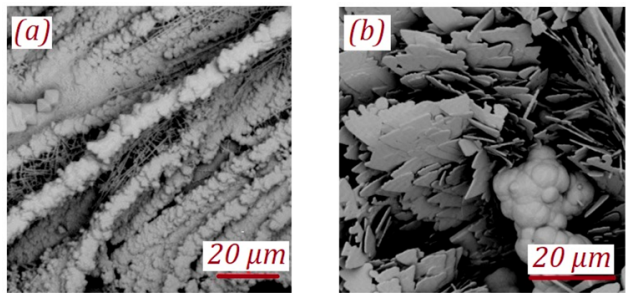

Figure 9. Detailed SEM views of $\mathrm{NaCl}$ crystals obtained from a saturated brine solution containing (a) 2\% (w/w) NTAA, (b) 4\% (w/w) NTAA.

planar surface are retarded (I and II in Figure 10) by lowering the supersaturation and by increasing amounts of adsorbed additive

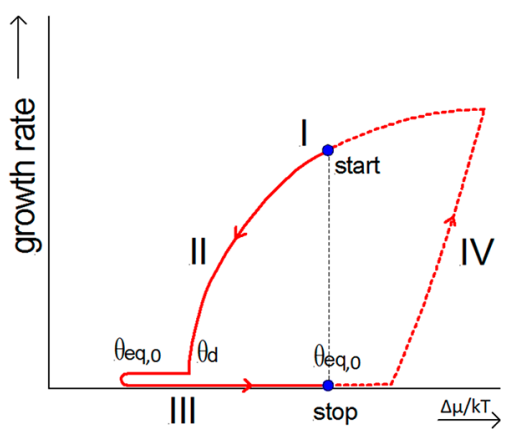

Figure 10. Hysteresis in the dependence of growth rate on supersaturation, induced by additive adsorption.

until the advancement of steps is completely blocked. This happens above a critical additive surface coverage of

$$
\theta_{\mathrm{d}} \propto\left(\frac{1}{d^{2}}\right)^{*} \cong\left[\frac{(\Delta \mu / k T) h_{\mathrm{st}}}{1.51 \gamma \Omega}\right]^{2}
$$

in which $d=$ average distance between adsorbed additives, $\Delta \mu$ / $k T$ is supersaturation, $h_{\text {st }}=$ step height, $\gamma$ is step free energy, and $\Omega$ the volume of one growth unit. ${ }^{31}$ As now no further growth occurs, more impurities accumulate until the equilibrium concentration at zero growth rate $\theta_{\mathrm{eq}, 0}>\theta_{\mathrm{d}}$ is attained (III in Figure 10). If the supersaturation is increased to such an extent that $\theta_{\mathrm{d}}>\theta_{\mathrm{eq}, 0}$, then growth restarts and growth steps pass the impurity fence "cleaning" the surface. On this surface freed from additives steps can propagate again, also for lower supersaturations (IV in Figure 10). A similar process occurs for roughened crystal faces as exemplified in ref 29. On these thermally/kinetically roughened F-faces or $\mathrm{S}$ and $\mathrm{K}$ faces, growth is no longer determined by step flow, but by a more or less random addition and removal of growth units. ${ }^{32}$ In this case $\theta_{\mathrm{d}}$ is determined by the surface free energy of the crystal-additive molecule boundary and supersaturation. ${ }^{29}$ In both situations, a hysteresis in the growth rate versus supersaturation curve can occur as shown in Figure 10.

In our model we consider the $3 \mathrm{D}$ growth of needle crystals, the nonfaceted tip surface of which is roughened by additive adsorption and fast growth. The needle side faces tend to be faceted, so here growth likely involves steps. Figure 11 shows the
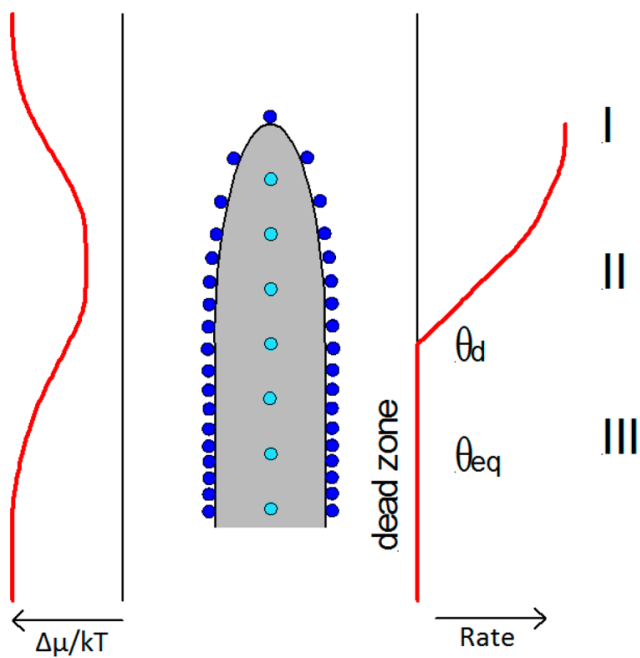

Figure 11. Mechanism of additive induced $\mathrm{NaCl}$ needle growth.

principle of the model, using the ideas presented in Figure 10. We consider three successive stages: (I) Fast growth of the tip region as a consequence of morphological instability induced by high supersaturation and lowering of surface free energy, which is typical for additive induced creeping processes. ${ }^{23}$ Here, growth is kinetically roughened and largely limited by mass transport. The higher growth rate leads to an enhanced incorporation of additives into the crystal lattice, which again results in a lower additive surface coverage as explained in the next section. In region (II), the interfacial supersaturation decreases as a consequence of an expansion of the solute diffusion field, typical for mass transport limited growth of dendritic or needle crystals. ${ }^{33}$ This leads to a decrease in growth rate, resulting in an increase in additive surface coverage which reinforces this decrease in growth rate. At a given moment we enter region (III) where the critical surface coverage, $\theta_{\mathrm{d}}$ is reached and growth stops. Here more and more additives will be adsorbed at the surface until the equilibrium concentration, $\theta_{\mathrm{eq}, 0}$, is achieved. As a consequence of the halt of growth, no mass transport is needed, and the interfacial supersaturation of growth units increases to the bulk value. However, in our case the bulk supersaturation is insufficient to break through the impurity fence, restarting 
growth. Growth remains blocked and stage IV in Figure 10 is never attained in our case. As growth in the region behind the tip is completely blocked, no side branching occurs and needles rather than dendrites are formed. For GLDA as an additive, an onset of dendritic growth can be perceived (Figure 7e,f).

Inspired by the work of Land et al., ${ }^{27}$ theoretical graphs of the growth rate with respect to the supersaturation are shown in Figure 12. These graphs help to understand the crystal growth
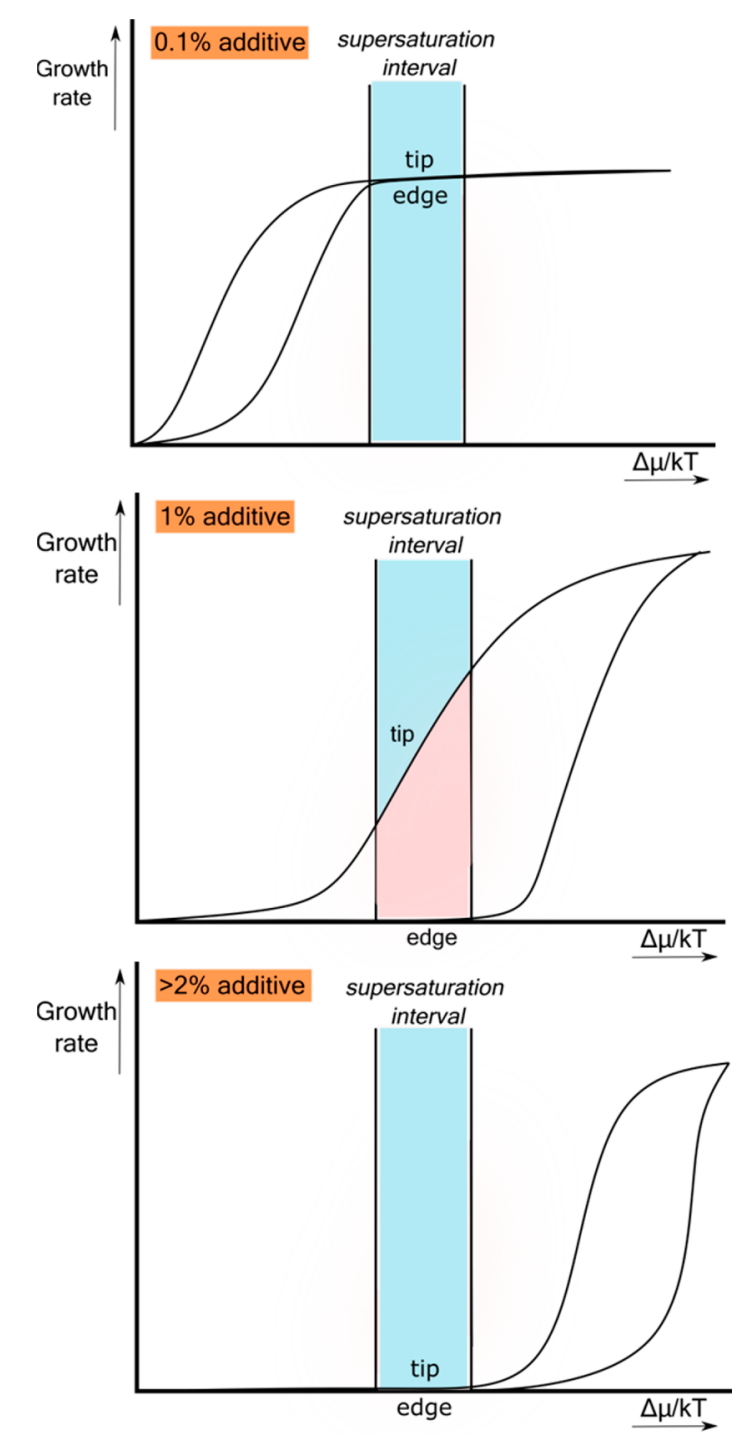

Figure 12. Growth rate curves describing the case of $0.1 \%(\mathrm{w} / \mathrm{w}), 1 \%$ $(\mathrm{w} / \mathrm{w})$, and $>2 \%(\mathrm{w} / \mathrm{w})$ additive. The supersaturation regime during the droplet evaporation is indicated in blue in the diagram, with the pink section indicating the presence of needle growth. In the cases of $0.1 \%$ $(\mathrm{w} / \mathrm{w})$ and $>2 \%(\mathrm{w} / \mathrm{w})$ additive, there is no needle growth, as the growth rates of the tip and edge are the same or almost zero at this specific supersaturation interval.

phenomena that we observed for different additive concentrations in this investigation. As mentioned before and described in refs 27-29, additives cause a hysteresis in crystal growth. At low supersaturations, growth is blocked; at a specific supersaturation the "dead zone" of no-growth is overcome and crystal growth sets in. Increasing the supersaturation further and then lowering it again leads to continued growth, also in the "dead zone" region, until it stops at a considerably lower super- saturation. The hysteresis interval with the "dual" growth rate shifts to higher supersaturation values for increased additive concentration, as displayed in Figure 12.

The impurity induced hysteresis in growth rate versus supersaturation explains the additives concentration dependence of needle formation. This is exemplified in Figure 12, which schematizes the "two-fold" growth rate versus supersaturation curves for different additive concentrations. The supersaturation interval used in the droplet experiments is indicated in blue and pink, the latter for the region where needles are formed. For the lowest additive concentration (in our case $0.1 \%(\mathrm{w} / \mathrm{w})$ ), the growth of the needle side faces is not blocked and cubic crystals are formed. For medium additive concentrations (0.75-1.5\% $(\mathrm{w} / \mathrm{w}))$ the needle tip grows, while the side faces are blocked. For the highest additive concentrations, exceeding $2 \%$ (w/w), also tip growth is blocked and featureless crystals rather than needles are formed. One should realize that supersaturation of the droplet experiments increases for increasing additive content to some extent, but this does not alter the general conclusions.

Additive Surface Coverage As a Function of Crystal Growth Rate in Needle Growth. As an approximation we consider a needle tip as a parabola $y(x)=a x^{2}$ advancing in the $-y$ direction at a constant rate $v_{\text {tip }}$, as schematized in Figure 13. The
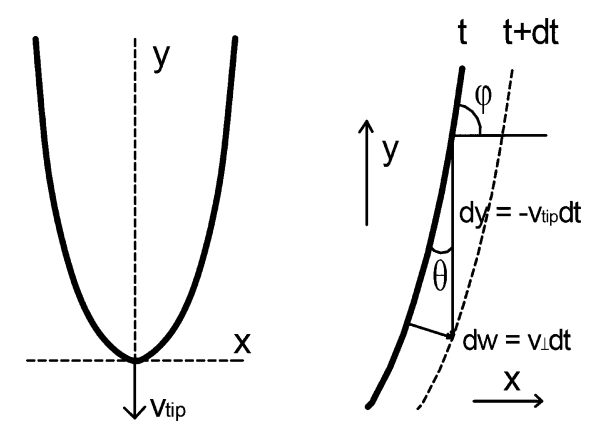

Figure 13. Perpendicular growth rate, $v_{\perp}$, as a function of the distance, $y$, from a parabolic needle tip.

ratio of needle growth perpendicular to its surface, $\mathrm{d} w$, and the needle advancement in the $-y$ direction, $d y$, is

$$
-\frac{\mathrm{d} w}{\mathrm{~d} y}=\frac{v_{\perp} \mathrm{d} t}{v_{\text {tip }} \mathrm{d} t}=\frac{v_{\perp}}{v_{\text {tip }}}=\sin (\theta)
$$

as follows from Figure 13. As the parabola slope angle

$$
\varphi=\arctan \left(\frac{\mathrm{d} y(x)}{\mathrm{d} x}\right)=\arctan (2 a x)=\arctan \left(2 a^{1 / 2} y^{1 / 2}\right)
$$

and $\theta=90^{\circ}-\varphi$, it follows that

$$
v_{\perp}=v_{\text {tip }} \cos (\varphi)=v_{\text {tip }} \cos \left(\arctan \left(2 a^{1 / 2} y^{1 / 2}\right)\right)
$$

It is clear that the perpendicular growth rapidly decreases further away from the needle tip (i.e., for increasing $y$ ).

To keep matters simple, we presume equilibrium surface coverage of the additives during needle growth. Following the Langmuir's adsorption isotherm, this equilibrium surface coverage is given by

$$
\theta_{\mathrm{eq}}=\frac{k_{\mathrm{a}} c}{k_{\mathrm{a}} c+k_{\mathrm{d}}}
$$

with $k_{\mathrm{a}}$ the adsorption rate per surface site. The other term 


$$
k_{\mathrm{d}}=k_{\mathrm{d}}^{0}+b R^{n}
$$

is the rate constant of removal of adsorbed additive units per surface site. In this, $k_{\mathrm{d}}^{0}$ is the rate constant of desorption of additive molecules and $b R^{n}$ is the rate constant of incorporation of additives into the crystal lattice, which we presume to be an exponential function of the growth rate, or $\left(v_{\perp}\right)^{n}$, Further, $b$ is a constant. From the growth rate dependence of $\theta_{\text {eq }}$,

$$
\theta_{\text {eq }}=\frac{k_{\mathrm{a}} c}{k_{\mathrm{a}} c+k_{\mathrm{d}}^{0}+b v_{\perp}^{n}}
$$

it follows that the additive coverage on the growing needle faces increases with distance $y$ from the needle tip according to

$$
\theta_{\text {eq }}(y)=\frac{k_{\mathrm{a}} c}{k_{\mathrm{a}} c+k_{\mathrm{d}}^{0}+b\left[v_{\text {tip }} \cos \left(\arctan \left(2 a^{1 / 2} y^{1 / 2}\right)\right)\right]^{n}}
$$

For $y=0$,

$$
\theta_{\text {eq }}(0)=\frac{k_{\mathrm{a}} c}{k_{\mathrm{a}} c+k_{\mathrm{d}}^{0}+b v_{\text {tip }}^{n}}
$$

the surface coverage is low and many additives are incorporated in the lattice. For $y=\infty$ or if $v_{\perp}=0$

$$
\theta_{\mathrm{eq}}(\infty)=\theta_{\mathrm{eq}, 0}=\frac{k_{\mathrm{a}} c}{k_{\mathrm{a}} c+k_{\mathrm{d}}^{0}}
$$

We can see that $\theta_{\text {eq }}$ increases for increasing $y$ until it reaches the value of $\theta_{\mathrm{d}}$ and growth stops, i.e., $v_{\perp}=0$. Then, more additives accumulate at the surface until the situation given by eq 10 ; i.e., a surface coverage of $\theta_{\text {eq, } 0}$, is reached. Then, growth is irreversibly blocked for the supersaturations applied in our case. Partial incorporation of additives is essential in our model, as the value of $\theta_{\text {eq }}(\infty)$ is attained readily if $b=0$, blocking growth completely and no crystals will be formed.

\section{CONCLUSIONS}

During this investigation, we have observed that when concentrations of $\sim 1 \%(\mathrm{w} / \mathrm{w})$ nitrilotriacetamide, methylglycine diacetamide, and glutamic acid diacetamide are added to a saturated brine solution and allowed to evaporate, a dense layer of ultrathin crystal needles, $0.3-2 \mu \mathrm{m}$ wide, is formed.

We concluded experimentally that these needles are formed from cubic $\mathrm{NaCl}$, with no evidence of large amounts of amide in the composition. We also determined that the crystallographic direction of the needles is $\langle 100\rangle$. Overall however, there is no preferred direction in which the needles grow on the substrate, leading to a chaotic "criss-cross" pattern.

From high magnification SEM observation, we concluded that the needle shape of these crystals is not due to parallel dislocations or twinning. We interpreted the $\mathrm{NaCl}$ needle growth by tip formation induced by morphological instability at the earliest stage followed by additive adsorption on the needle side faces, preventing thickness growth and side branch formation. Needle formation in this work is a unique combination of initial morphological instability and subsequent additive blocking of crystal growth.

\section{ASSOCIATED CONTENT}

\section{S Supporting Information}

The Supporting Information is available free of charge on the ACS Publications website at DOI: 10.1021/acs.cgd.7b01170.
Movie S1: High magnification optical microscope movie showing the propagation of ultrathin needle growth from a saturated brine solution containing $1 \%(\mathrm{w} / \mathrm{w})$ NTAA (AVI)

\section{Accession Codes}

CCDC 1533348-1533349 contain the supplementary crystallographic data for this paper. These data can be obtained free of charge via www.ccdc.cam.ac.uk/data_request/cif, or by emailing data_request@ccdc.cam.ac.uk, or by contacting The Cambridge Crystallographic Data Centre, 12 Union Road, Cambridge CB2 1EZ, UK; fax: +44 1223336033.

\section{AUTHOR INFORMATION}

\section{Corresponding Author}

*E-mail: w.vanenckevort@science.ru.nl. Tel: +31 (0)24 365 3433. Fax: +31 (0)243653067.

\section{ORCID}

Willem J. P. van Enckevort: 0000-0001-7436-8391

Elias Vlieg: 0000-0002-1343-4102

\section{Notes}

The authors declare no competing financial interest.

\section{ACKNOWLEDGMENTS}

We would like to acknowledge AkzoNobel Industrial Chemicals for the funding of this project.

\section{REFERENCES}

(1) de Romé de L'Isle, J. B. L. Cristallographie ou description des formes propres à tous les corps du regne minéral, dans l'état de combinaison saline, pierreuse ou métalliques; Imprimerie du Monsieur: Paris, 1783; p 379.

(2) Fenimore, C.; Thraillkill, A. J. Am. Chem. Soc. 1949, 71, 27142717.

(3) Royer, L. Compt. Rend. 1934, 198, 585.

(4) Gille, F.; Spangenberg, K. Z. Kristallogr. - Cryst. Mater. 1927, 65, 204.

(5) Retgers, J. W. Z. Phys. Chem. 1892, 9, 267.

(6) Radenovic, N.; Kaminski, D.; van Enckevort, W. J. P.; Graswinckel, S.; Shah, I.; in 't Veld, M.; Algra, R.; Vlieg, E. J. Chem. Phys. 2006, 124, 164706.

(7) Radenovic, N.; van Enckevort, W. J. P.; Verwer, P.; Vlieg, E. Surf. Sci. 2003, 523, 307-315.

(8) Scott, T. R., Bromby, N. G., Allday, C. U.S. Patent, 2990246, 1961.

(9) Phoenix, L. Br. Chem. Eng. 1966, 11, 34-38.

(10) Sarig, S.; Glasner, A.; Epstein, J. A. J. Cryst. Growth 1975, 28, 295299.

(11) Sarig, S.; Tartakovsky, F. J. Cryst. Growth 1975, 28, 300-355.

(12) Boone, D. E., Ralston, P. H. U.S. Patent, 3544461, 1970.

(13) Kelland, M. A. Production Chemicals for the Oil and Gas Industry; CRC Press, 2009; p 74-75.

(14) Tauber, H.; Kleiner, I. J. Am. Chem. Soc. 1932, 54, 2392-2393.

(15) Hinegardner, W. S. J. Am. Chem. Soc. 1933, 55, 1461-1462.

(16) Sears, G. W. J. Chem. Phys. 1957, 26, 1549.

(17) Shichiri, T.; Kato, N. Acta Metall. 1965, 13, 785-795.

(18) Shichiri, T.; Kato, N. J. Cryst. Growth 1968, 3-4, 384-390.

(19) Shichiri, T. J. Cryst. Growth 1974, 24-25, 350-353.

(20) van Damme-van Weele, M. A. Adsorption et Croissance Cristalline, Centre National de la Recherche Scientifique, Paris, 1965.

(21) Noorduin, W., private communication.

(22) Townsend, E. R.; Swennenhuis, F.; van Enckevort, W. J. P.; Meijer, J. A. M.; Vlieg, E. CrystEngComm 2016, 18, 6176-6183.

(23) Townsend, E. R.; van Enckevort, W. J. P.; Meijer, J. A. M.; Vlieg, E. Cryst. Growth Des. 2017, 17, 3107-3115.

(24) Wagner, R. S. Acta Metall. 1960, 8, 57.

(25) Hamilton, D. R.; Seidensticker, R. G. J. Appl. Phys. 1960, 31, 1165. 
(26) van Enckevort, W. J. P.; Graef, M. W. M. J. Electrochem. Soc. 1981, $128,154-161$.

(27) Land, T. A.; Martin, T. L.; Potapenko, S.; Tayhas Palmore, G.; de Yoreo, J. J. Nature 1999, 399, 442-445.

(28) Miura, H. Cryst. Growth Des. 2016, 16, 2033-2039.

(29) van Enckevort, W. J. P.; van den Berg, A. C. J. F. J. Cryst. Growth 1998, 183, 441-445.

(30) Cabrera, N.; Vermilyea, D. A. Growth and Perfection of Crystals; Wiley: New York, 1958; pp 393-410.

(31) Potapenko, S. Y. J. Cryst. Growth 1993, 133, 147-154.

(32) Bennema, P. In Growth and Morphology of Crystals, Handbook of Crystal Growth; Hurle, D.T.J., Ed.; Elsevier Science Publishers: Amsterdam, 1993; Vol. 1, Chapter 6, pp 477-581.

(33) Glicksman, M. E.; Marsh, S. P. The Dendrite. In Handbook of Crystal Growth; Hurtle, D. T. J. Ed.; Elsevier Science Publishers: Amsterdam, 1993; Vol. 1b, Chapter 15, pp 1075-1122. 